\title{
Probabilidad más que solo suerte
}

\author{
Gabriel Mena C. \\ gabriel.mcantero@outlook.com \\ Universidad de Costa rica
}

\author{
Andrea Navarro A. \\ andre_na0393@hotmail.com \\ Universidad de Costa rica
}

Recibido: Setiembre 1, 2016

Aceptado: Noviembre 15, 2016

Resumen. Este taller va dirigido a profesores y estudiantes universitarios de Enseñanza de la Matemática que deseen capacitarse en el tema de las probabilidades para poder ampliar sus conocimientos e impartir con mayor seguridad las clases de este tema, buscando incentivar el interés de sus estudiantes. El principal objetivo del taller es estudiar la relación que existe entre las eventualidades equiprobables, la Ley de Laplace y muchas situaciones o experimentos básicos de la teoría de las probabilidades.

Utilizando lo planteado por los Programas de Matemática del Ministerio de Educación Pública, se procurará generar un ambiente de construcción del conocimiento, utilizando para esto la integración de la historia con problemas importantes en la teoría de las probabilidades, la elaboración de materiales simples con los cuales modelar las situaciones planteadas, el uso de elementos del entorno para la modelización de fenómenos probabilísticos y se incluirá un módulo de uso del programa Excel como modelador de situaciones, en el que se pueda establecer la relación entre probabilidad frecuencial y probabilidad total, especialmente en experimentos con eventualidades equiprobables.

Palabras clave: Probabilidad, aleatoriedad, Ley de Laplace, docentes, recursos.

Abstract. This workshop is oriented to teachers and college students primarily of Math Teaching, who wants to expand their knowledge in probability and train in different ways to attract interest and focus of students in the classroom, and also been more secure when imparting probability lessons. The main goal of the workshop is to study the relation between equiprobables eventualities, Laplace's Law, and many situations and experiments in probability theory.

Using what states in the Mathematical Programs of the Public Education Ministry, it'll be the intention to generate a suitable environment for the construction of knowledge, using the history through historical problems in the probability theory, the elaboration of simple and handy materials to model given situations, the use of environmental materials in order to model probabilistic situations, and also there will be a module of the program Excel as a way to model different situations and also to relate frequency probability with total probability, especially in experiments with equiprobable eventualities. 
KeyWords: Probability, randomness, Laplace’s Law, teachers, resources

\subsection{Introducción}

Mediante un taller de aproximadamente 4.5 horas se pretende construir o reconstruir los diferentes conceptos probabilísticos asociados a la Ley de Laplace, que corresponden a los contenidos y habilidades presentes en el Programa de Estudios de Matemática para octavo año.

El taller se presenta en tres sesiones de 1.5 horas cada una, realizadas en tres días diferentes, en la primera sesión se busca la consolidación de los diferentes conceptos para ser puestos en práctica mediante el análisis pedagógico y didáctico de los mismos.

En la segunda sesión se propicia el espacio para la construcción de situaciones probabilísticas que involucren la Ley de Laplace, para analizar desde los puntos de vista de los participantes los diferentes ejercicios propuestos. Finalmente, para el último día de taller se modelan diferentes situaciones probabilísticas también relacionadas con la Ley de Laplace, mediante el programa Excel que permite acceder al uso de las TIC's como una herramienta de aprendizaje.

\subsection{Justificación}

Al enfrentar los recientes cambios presentes en el programa de matemáticas de la educación costarricense, los docentes han manifestado diferentes deficiencias no solo en la formación académica referente específicamente a Probabilidad y Estadística, sino también en las decisiones pedagógicas tomadas en su planeamiento y en especial en las diversas prácticas didácticas realizadas en clase.

Referente a ésta situación, Pérez, Cueto, Fernández, Filloy, Diez, Kelmansky \& Pomilio en su trabajo Mejorando Las Competencias Para La Enseñanza De La Estadística De Profesores De Secundaria En Formación A Través De Talleres Participativos para las memorias de IASE 2015 Satellite Paper - Refereed, señalan:

"Esta escasa preparación en la disciplina con la que el profesor termina sus estudios hace que cuente con pocos recursos para su enseñanza y evidencia una actitud negativa hacia la disciplina. En ese sentido, Estrada (2004) halló que la actitud hacia la estadística del profesor en ejercicio se deteriora con la práctica docente, debido a la dificultad que él mismo encuentra en la disciplina, a la escasa importancia que se le otorga o a la dificultad para aprender que aprecia en sus alumnos. Como consecuencia, los contenidos de estadística no son habitualmente desarrollados en el ciclo lectivo o bien quedan reducidos a unas pocas clases, con un tratamiento habitualmente limitado a los aspectos procedimentales." (Pérez, Cueto, Fernández, Filloy, Diez, Kelmansky \& Pomilio. 2015 [1])

En nuestro país la situación de la enseñanza de la Estadística y la Probabilidad enfrenta los mismos problemas que se señalan anteriormente, problemas que condicionan el aprendizaje de los estudiantes 
a procedimientos para obtener simplemente resultados numéricos y no a la interiorización de los conceptos para propiciar un análisis de significados, que es lo que busca tanto el Programa de Estudios de Matemática de secundaria para la resolución de problemas de la cotidianidad, como las disciplinas matemáticas de Estadística y Probabilidad.

Es por esto que no se está logrando el objetivo de modelar la realidad inmediata de los estudiantes mediante la Estadística y la Probabilidad y de esta manera acercar la matemática al estudiante y contextualizarla, se reducen las disciplinas y sus saberes a meros cálculos, algoritmos y números.

En relación con, esto Milagros Rodríguez señala en su artículo El Perfil Del Docente De Matemática: Visión Desde La Triada Matemática-Cotidianidad Y Pedagogía Integral para la revista Electrónica Actualidades Investigativas en Educación (Redalyc) menciona:

"Entonces, como ha desaparecido el diálogo en el acto de enseñar el proceso de enseñanza de la Matemática se ha simplificado y se remite al dictado de una teoría ya acabada, donde el estudiante no inmiscuye su cotidianidad, su cultura, sus sentimientos." (Rodríguez 2010 [2], pág 4)

Desligar el dialogo que debe existir entre la escuela y el contexto limita la oportunidad del estudiante de aprender el concepto matemático, lo que genera no solo deficiencias en el desarrollo de la clase y la resolución de ejercicios sino se pierde la matemática como una herramienta útil para otras disciplinas del saber.

Con estas situaciones que acompañan a la Enseñanza de la Estadística y la Probabilidad se propone un taller dirigido a fortalecer los diferentes conceptos probabilísticos asociados al estudio de la Ley de Laplace en docentes de Matemática; para impartir en octavo grado de secundaria, el cual, será complementado con una sección de tecnología mediante el uso de Excel.

\section{Taller}

\section{Día I}

Tiempo: 1 hora y 30 minutos

Materiales: Juego de mesa, en este caso Pictionary, Pizarra, Marcadores.

Luego de una presentación sencilla por parte de cada uno de los participantes, se procederá a realizar una actividad rompehielos, que será una adaptación del juego Pictionary, el cual consiste en lanzar un dado cuyas caras serán de seis colores, amarillo, rojo, verde, naranja , azul y morado. Los primeros 5 colores corresponden a las categorías que aparecen en cada una de tarjetas, las cuales que tienen escrita una palabra que será representada en la pizarra mediante un dibujo, el sexto color, el morado, indicará que el participante puede escoger la opción que prefiera de las palabras que aparecen en la tarjeta. Un segundo dado tendrá en cuatro de las caras una dificultad, dibujar con la mano débil, dibujar con los ojos cerrados, no levantar el lápiz del papel, hacer dos dibujos, las otras dos caras son para dibujar sin dificultad. Finalmente, se procederá a dibujar en una pizarra la palabra que se obtiene de la tarjeta, con la dificultad obtenida, tratando que los compañeros adivinen de qué se trata. Esto con el fin de que comiencen a familiarizarse con el tipo de actividades que se pueden realizar para 
ejemplificar situaciones probabilísticas. Después de que todos o la mayoría de los participantes hayan dibujado al menos una vez, se procederá a discutir los resultados obtenidos en los dados y los dibujos, llegando a la conclusión de que existe lo que se puede denominar un "espacio de posibles resultados" para cada uno de estos, llegando así a la construcción empírica del concepto de espacio muestral, eventualidad y evento. Seguidamente, se procederá a discutir las nociones de aleatoriedad, probabilidad y determinismo, mediante la conversación y la lluvia de ideas.

Seguidamente se presenta una actividad en la que se proponen tres situaciones clásicas de la teoría de las probabilidades

- Los jugadores A y B apuestan a cara o cruz, tirando una moneda. El jugador que primero llega a cinco puntos gana la apuesta. El juego se interrumpe en un momento en que A tiene 4 puntos y B tiene 3 puntos. ¿Cómo deben repartir la cantidad apostada para ser justos? ¿la proporción justa es de 4 a 3 ?

- El Caballero De Meré sabía que era ventajoso apostar por el resultado de obtener al menos un seis en una serie de 4 lanzamientos de un dado. Entonces De Meré argumentó que debiera ser igualmente ventajoso apostar por el resultado de obtener al menos un doble seis en una serie de 24 lanzamientos con un par de dados. Para ello había razonado "por regla de tres": si en 4 lanzamientos se apuesta por un resultado específico entre 6 posibles, es lo mismo que si en 24 lanzamientos se apuesta por un resultado específico entre 36 posibles, ya que $6: 4=36: 24$. La experiencia no corroboró la suposición de De Meré. ¿cómo hizo Pascal, justificar por qué la primera apuesta es ventajosa pero la segunda no lo es?

- Tres jugadores A, B y C meten 12 fichas en una bolsa de las que 4 son blancas y 8 negras. El ganador es quién primero extraiga una ficha blanca. Primero extrae A, luego B y luego C, y el ciclo se vuelve a iniciar hasta que alguno gane. Qué relación hay entre las probabilidades de ganar que tiene cada jugador si: a) hay solo una bolsa y cada vez que se extrae una ficha negra se devuelve a la bolsa; b) las extracciones son sin reemplazamiento; c) cada uno de los tres jugadores comienza con su propia bolsa de doce fichas y las va extrayendo sin reemplazamiento.

En cinco grupos los participantes deberán contestar las preguntas de cada ítem y presentar sus razonamientos probabilísticos del porqué de sus respuestas.

Esta situación anterior es de suma importancia, ya que además de integrar la historia al proceso didáctico, permite ver errores de preconcepciones y nociones que se tiene en la realidad. Así mismo, este ejercicio permite desarrollar más a fondo el concepto de espacio muestral, evento, eventualidad, además de permitir introducir los conceptos de eventualidades equiprobables, Ley de Laplace, probabilidad frecuencial, probabilidad teórica y la Ley de los Grandes Números. Lo anterior dado que al buscar y razonar las respuestas se puede comprender de mejor manera cada concepto y verificar que se esté adquiriendo correctamente cada uno. Se discutirá cada situación y sus principales características. 
Día II

Tiempo: 1 hora y $\mathbf{3 0}$ minutos

Materiales: Goma, tijeras, lápices, marcadores, cinta adhesiva, papeles de colores, cartulinas, paletas, silicón frío.

Se retomarán algunos de los conceptos adquiridos en el día anterior mediante el repaso.

Se le solicita a los participantes que, en grupos de tres personas, redacten una situación probabilística, con los conceptos que poseen de la teoría de las probabilidades, pueden usar si gustan sus teléfonos móviles o computadoras, para obtener ayudas, sin embargo se solicita que sea una trabajo original, y que no requiera de material digital para su solución, por el contrario debe realizarse con material de reciclaje cada uno de los materiales didácticos que modelan la situación tales como, dados utilizando paletas o palitos de madera y cinta adhesiva o papel y goma, monedas usando cartón, urnas con bolas utilizando canicas, o cualquier método que utilice materiales de reciclaje y que conozcan cómo hacerlos o encuentren su forma de confección con ayuda de internet. La idea es prescindir de los materiales digitales, ya que no todos los centros educativos cuentan con éstos recursos para cada estudiante, dicha situación será expuesta con su respectiva solución, objetivo específico dentro del contenido de Ley de Laplace; materiales necesarios para su realización y cómo se construye, tiempo esperado de solución, pros y contras del ejercicio y posibles errores que pueden cometer los estudiantes, se les pedirá que sean lo más específicos posibles. Esto con el fin de que el taller no se quede en el conocimiento teórico, sino que se lleve al ambiente práctico y se incentive al docente a buscar situaciones que pueda utilizar en el aula.

Seguidamente se realizará la exposición de los ejercicios planteados por cada uno de los grupos. Se recomienda a los demás participantes que tomen notas de cada una de las situaciones, sobre qué les llamó la atención, qué creen que se debe mejorar y qué cambiarían de acuerdo a su contexto. Para finalmente, producir una discusión acerca de lo expuesto por cada uno con el fin de mejorar las actividades y realizar un abordaje y planeamiento en conjunto, tratando así de expandir las opciones y posibilidades para la realización de actividades en el aula por los participantes y notando que todas estas propuestas son adaptables al contexto de cada uno.

\section{Día III}

Tiempo: 1 hora y $\mathbf{3 0}$ minutos

Materiales: Computadora y el programa EXCEL.

Para este último día se realizará un repaso de la teoría que ha visto en los últimos días, esto con el fin de que los conceptos y conocimientos sean interiorizados y manejados de la mejor manera por parte de los participantes.

Seguidamente, se procederá a trabajar con el programa Excel, para lo cual es necesario que se comience con una introducción al uso de las funciones básicas de programación que tiene este programa, como SI, CONTAR, CONTAR.SI, RAND, entre otras que se consideren necesarias.

A continuación, se demostrará cómo es que se puede modelar una situación probabilística básica como el lanzamiento de un dado en este programa y al finalizar esto se procederá a 
explicar cómo se puede programar el conteo de los resultados de los posibles eventos.

Se modelarán los siguientes experimentos cuyo grado de dificultad es creciente.

- Si se lanza un dado legal, ¿cuál es la probabilidad de que salga un múltiplo de 2?

- Si se lanzan dos dados legales ¿cuál es la probabilidad de que la suma de los resultados sea menor a 8 ?

- Si se lanzan 3 monedas al mismo tiempo, ¿cuál es la posibilidad de que salgan por lo menos 2 escudos?

Y para finalizar, se propondrá comparar la probabilidad frecuencial de que suceda un evento de los que fueron programados anteriormente en forma escalada de 10, 100, 1000, 3000 y 5000 repeticiones del mismo, y la probabilidad teórica de que suceda, esto con el fin de que se evidencie cómo a mayor cantidad de repeticiones la probabilidad frecuencial y la teórica se van acercando, siendo esto una manifestación de lo planteado por la Ley de los Grandes Números.

\subsection{Conclusiones}

El desarrollar un taller para la construcción y análisis de los diferentes conceptos de probabilidad asociados a la Ley de Laplace, propicia un espacio que permita facilitar ideas que los participantes pueden poner en práctica en una clase de Estadística y Probabilidad en secundaria. Además, se cuenta con un espacio donde se puede construir, conversar y analizar desde diferentes puntos de vista los distintos conceptos y ejercicios propuestos para corregir posibles errores y mejorar el ambiente del aula, haciéndolo más interesante y atractivo para los estudiantes, propiciando así un aprendizaje significativo y participativo.

Los diferentes problemas propuestos en el taller, para el análisis del razonamiento, posibles errores y aciertos, son situaciones que propiciaron el avance en el pensamiento probabilístico a lo largo de la historia, de manera, el taller incluye la historia como parte de la construcción que se propone buscando un desarrollo integral de los diferentes conceptos que se buscan fortalecer y promoviendo el uso de la misma como una poderosa herramienta en el quehacer docente, especialmente en matemática.

El trabajo de construir con materiales de reciclaje diferentes objetos que permitan modelar y ejemplificar las diferentes situaciones y problemas de probabilidad que pueden resolverse en una clase de secundaria permite a los participantes reflexionar sobre lo que se tiene como recursos y no sobre las carencias que se le presentan promoviendo así la proactividad docente, de manera que es posible un trabajo creativo y eficiente que permita a los estudiantes de secundaria obtener mediante diferentes medios los conocimientos que se requieren, sin tener que recurrir exclusivamente a herramientas tec- 
nológicas u otro material didáctico muchas veces inaccesible para el estudiantado por su costo.

El uso de las tecnologías dentro de nuestro sistema educativo es un tema del cual no se puede prescindir dentro de las conversaciones de didáctica de la matemática, ya que en una cultura globalizada como la que vivimos, se busca relacionar cada aspecto de nuestra vida con objetos que faciliten nuestras tareas, de esta manera nuestros estudiantes se ven influenciados por los diferentes aparatos tecnológicos que tienen a su alcance. Para los que sí los tienen, pues no es posible generalizar la presencia de dichos recursos en todas las instituciones educativas y para todos los estudiantes por igual, se busca, mediante el taller, modelar diferentes situaciones probabilísticas que permitan a los docentes crear una nueva perspectiva que puedan desarrollar en su clase de probabilidad en secundaria, logrando de esta manera acercar al estudiante a los conceptos y ofreciéndole una perspectiva diferente y novedosa de aprender, la cual se ajusta a sus intereses y facilidades, potenciando el aprendizaje.

\section{Bibliografía}

[1] Pérez. A, Cueto. G, Fernández. M, Filloy. J, Diez. S, Kelmansky.D \& Pomilio. C. (2015) “Mejorando Las Competencias Para La Enseñanza De La Estadística De Profesores De Secundaria En Formación A Través De Talleres Participativos". Memorias de IASE Satellite Paper - Refereed. Argentina.

[2] Rodríguez. M. (2010). “El Perfil Del Docente De Matemática: Visión Desde La Triada MatemáticaCotidianidad Y Pedagogía Integral”. Revista Electrónica Actualidades Investigativas en Educación. Vol. 10, Núm. 3. DOI: http://dx.doi.org/10.15517/aie.v10i3.10150 